\title{
On Omega Index and Average Degree of Graphs
}

\author{
Sadik Delen $\mathbb{D}^{1},{ }^{1}$ Musa Demirci $\mathbb{D}^{1},{ }^{1}$ Ahmet Sinan Cevik $\mathbb{D}^{2},{ }^{2}$ and Ismail Naci Cangul $\mathbb{D i}^{1}$ \\ ${ }^{1}$ Faculty of Arts and Science, Department of Mathematics, Bursa Uludag University, 16059 Bursa, Turkey \\ ${ }^{2}$ Faculty of Science, Department of Mathematics, Selcuk University, Konya, Turkey \\ Correspondence should be addressed to Ismail Naci Cangul; cangul@uludag.edu.tr
}

Received 16 January 2021; Revised 25 February 2021; Accepted 28 October 2021; Published 12 November 2021

Academic Editor: Stanislaw Migorski

Copyright (c) 2021 Sadik Delen et al. This is an open access article distributed under the Creative Commons Attribution License, which permits unrestricted use, distribution, and reproduction in any medium, provided the original work is properly cited.

Average degree of a graph is defined to be a graph invariant equal to the arithmetic mean of all vertex degrees and has many applications, especially in determining the irregularity degrees of networks and social sciences. In this study, some properties of average degree have been studied. Effect of vertex deletion on this degree has been determined and a new proof of the handshaking lemma has been given. Using a recently defined graph index called omega index, average degree of trees, unicyclic, bicyclic, and tricyclic graphs have been given, and these have been generalized to $k$-cyclic graphs. Also, the effect of edge deletion has been calculated. The average degree of some derived graphs and some graph operations have been determined.

\section{Introduction}

Let $G=(V, E)$ be a finite, undirected, and simple graph, having $|V|=n$ vertices and $|E|=m$ edges. For a vertex $v \in V$, the number of edges of $G$ meeting at $v$ is denoted by $d_{G}(v)$ or $d_{v}$ and known as the degree of $v$. If the set of all vertex degrees of $G$ is

$$
D=\left\{1^{\left(a_{1}\right)}, 2^{\left(a_{2}\right)}, 3^{\left(a_{3}\right)}, \ldots, \Delta^{\left(a_{\Delta}\right)}\right\}
$$

where $a_{i}$ s are nonnegative integers, then $D$ is called the degree sequence of $G$. Here, $\Delta$ is the maximum vertex degree. For every graph $G$, there is a degree sequence. However, for a degree sequence, there may or may not exist a graph. If there is a graph, then $D$ is said to be realizable. For every realizable degree sequence, there is at least one graph; usually, there are many.

In [1], a new graph index called $\Omega$ index was defined by

$$
\Omega(D)=\sum_{i=1}^{\Delta}(i-2) a_{i} .
$$

Several properties of $\Omega$ index were obtained in $[1,2]$.

There are some graph invariants related to the vertex degrees. The density of a graph $G$ measures how many edges are there in the set $E$ of edges compared to the maximum possible number of edges between the vertices of $G$. For a simple graph, the maximum number of edges is attained when the graph is complete. In this case, this number would be $n(n-1) / 2$. So, the density of $G$ is

$$
\rho(G)=\frac{2 m}{n(n-1)} \text {. }
$$

Clearly, the density increases proportionally with the number of edges. Therefore, amongst all connected simple graphs having $n$ vertices, trees have the lowest density and complete graph $K_{n}$ has the highest density which is 1 .

In this paper, we make use of $\Omega$ index to study the properties of average degree $\overline{d_{G}}$ of a graph $G$ which measures how many edges has $G$ compared to the number of vertices, that is,

$$
\overline{d_{G}}=\frac{2 m}{n}
$$

From the definitions, we can easily deduce the following relation between $\overline{d_{G}}$ and $\rho(G)$ :

$$
(n-1) \rho(G)=\overline{d_{G}} .
$$

Also, the following relations between the $\Omega$ index of a graph $G$ and density and average degree can be deduced as follows: 


$$
\begin{gathered}
\rho(G)=\frac{\Omega(G)+2 n}{n(n-1)}, \\
\overline{d_{G}}=\frac{\Omega(G)}{n}+2 .
\end{gathered}
$$

If all the vertices in a graph have the same degree, say $r$, the graph is said $r$-regular. Most of the graphs are not regular and some graph indices have been defined to measure the irregularity of a graph. The Bell index of a graph $G$ is defined by

$$
B(G)=\sum_{u \in V(G)}\left(d_{G}(u)-\frac{2 m}{n}\right)^{2},
$$

see [3], and the degree deviation index is defined by

$$
S(G)=\sum_{u \in V(G)}\left|d_{G}(u)-\frac{2 m}{n}\right|,
$$

In [4], the degree deviation is equal to the product of the order $n$ of $G$ with the discrepancy of the graph. Also, the Collatz-Sinogowitz index is defined by

$$
C S(G)=\lambda_{1}-\frac{2 m}{n}
$$

where $\lambda_{1}$ denotes the largest eigenvalue of the adjacency matrix [5]. Note that, in all these irregularity indices, the average degree of a graph is used. This makes the notion of the average degree of a graph important. In the recent paper [6], two new and structurally different irregularity indices IRA and IRB have been defined and compared with other existing irregularity measures. Naturally, this list can easily be extended to have new members of the family of irregularity indices.

The structure of this paper is planned as follows. In Section 2, two related notions, density and average degree, are studied and these two quantities are calculated for acyclic, unicyclic, bicyclic, tricyclic, and, in general, $k$-cyclic graphs. In Sections 3 and 4, the effects of vertex and edge deletion on average degree of a graph are formulized. In Sections 5 and 6, the average degree of some derived graphs and some binary graph operations are determined.

\section{Density, Average Degree, and Cyclicness}

A connected graph $G$ having no faces is called acyclic. A graph having at least one face is named as cyclic; especially as unicyclic, bicyclic, tricyclic, etc., according to the number of faces which is $1,2,3$, etc., respectively. Here, using the $\Omega$ index, graphs are classified according to their cyclicness, and for each case, the density of the graph is characterized as follows:

$$
\begin{aligned}
& \text { (i) If } G \text { is acyclic, then } \rho(G)=(2 / n) \\
& \text { (ii) If } G \text { is unicyclic, then } \rho(G)=(2 /(n-1)) \\
& \text { (iii) If } G \text { is bicyclic, then } \rho(G)=((2(n+1)) /(n(n-1))) \\
& \text { (iv) If } G \text { is tricyclic, then } \rho(G)=((2(n+2)) / \\
& (n(n-1)))
\end{aligned}
$$

(v) If $G$ is $k$-cyclic, for $k=0,1,2, \ldots$, then $\rho(G)=((2(n+k-1)) /(n(n-1)))$

Indeed, let $G$ be a connected $k$-cyclic graph. By Theorem 1 in $[1]$, we have $k=(\Omega(G) / 2)+1$, as $G$ is connected. Hence, $\Omega(G)=2(k-1)$, and by Corollary 3.4 in [1], we know that $m=n+k-1$.

The following is a similar result for an average degree. The average vertex degree of a connected $k$-cyclic graph is

$$
\overline{d_{G}}=\frac{2(n+k-1)}{n}
$$

As a result, connected acyclic, unicyclic, bicyclic, tricyclic, etc., graphs have average degrees $2(n-1) / n, 2$, $2(n+1) / n, 2(n+2) / n$, etc., respectively.

\section{Effect of Vertex Deletion on Average Degree}

Now, the effect of vertex deletion on average degree will be considered. Using the obtained formula successively, we give a result which helps to calculate the average degree of a large graph by means of the average degree of a much smaller graph. Let $G-v$ be the graph obtained by deleting the vertex $v$ together with $d_{v}$ edges incident to $v$. First, we have the following.

Theorem 1. The following recurrence relation holds:

$$
n \cdot \bar{d}_{G}-(n-1) \bar{d}_{G-v_{1}}=2 d_{G}\left(v_{1}\right) \text {. }
$$

Proof. Let $G$ be a connected simple graph of order $n$. Let $v_{1} \in V(G)$ have degree $d_{G}\left(v_{1}\right)$ in $G . G-v_{1}$ is the graph obtained by deleting the vertex $v_{1}$ together with $d_{v_{1}}$ edges incident to $v_{1}$. Hence, deleting $v_{1}$ from $G$ reduces the degree of each of the $d_{v_{1}}$ neighbours of $v_{1}$ by 1 . So, the deletion of $v_{1}$ from $G$ reduces the total vertex degree of $G$ by $2 d_{v_{1}}$. Hence,

$$
\bar{d}_{G-v_{1}}=\frac{\sum_{u \in V(G)} d_{u}-2 d_{v_{1}}}{n-1}=\frac{n \cdot \overline{d_{G}}-2 d_{v_{1}}}{n-1},
$$

which gives the result.

Applying Theorem 1 recursively, we can give another proof of the handshaking lemma by labeling the vertices $v_{1}, v_{2}, \cdots, v_{n}$ so that $d_{v_{1}} \leq d_{v_{2}} \leq d_{v_{n}}$.

Corollary 1. (handshaking lemma). In every graph, the sum of vertex degrees is equal to twice the number of edges, that is,

$$
\sum_{u \in V(G)} d_{u}=2 m
$$

Proof. Applying Theorem 1 successively to delete $v_{1}, v_{2}, \ldots, v_{n}$ from $G$, respectively, we get the following equalities: 


$$
\begin{aligned}
n \bar{d}_{G}-(n-1) \bar{d}_{G-v_{1}} & =2 d_{v_{1}} \\
(n-1) \bar{d}_{G-v_{1}}-(n-2) \bar{d}_{G\left\{v_{1}, v_{2}\right\}} & =2 d_{v_{2}} \\
(n-2) \bar{d}_{G\left\{v_{1}, v_{2}\right\}}-(n-3) \bar{d}_{G\left\{v_{1}, v_{2}, v_{3}\right\}} & =2 d_{v_{3}} \\
& \vdots \\
(n-(n-1)) \bar{d}_{G\left\{v_{1}, v_{2}, \ldots, v_{n-1}\right\}}-(n-n) \bar{d}_{G\left\{v_{1}, v_{2}, \ldots, v_{n}\right\}} & =2 d_{v_{n}} . j .
\end{aligned}
$$

Adding all these side by side, we obtain the result.

So, we deleted all the vertices one by one to prove handshaking lemma. What happens if we only delete some of the vertices? We answer this question in two steps.

Theorem 2. Let $G$ be a connected simple graph of order $n$, size $m$, and average degree $\overline{d_{G}}$. If $v_{1}, v_{2}, \ldots, v_{k}$ are pairwise nonadjacent vertices of $G$, then

$$
\bar{d}_{G\left\{v_{1}, v_{2}, \ldots, v_{k}\right\}}=2 \frac{m-\left(d_{1}+d_{2}+\cdots+d_{k}\right)}{n-k},
$$

where $d_{i}=d_{v_{i}}$ for $i=1,2, \ldots, k$.

Proof. Let $\bar{m}$ and $\bar{n}$ be the size and order of $G-\left\{v_{1}, v_{2}, \ldots, v_{k}\right\}$. Then, by definition, we have $\bar{d}_{G-\left\{v_{1}, v_{2}, \ldots, v_{k}\right\}}=2(\bar{m} / \bar{n})$. Clearly, $\bar{n}=n-k$ as $k$ vertices are deleted. When a vertex $v_{i}$ is deleted, note that $d_{i}$ incident edges are also deleted. So, if we delete pairwise nonadjacent vertices $v_{1}, \ldots, v_{k}$ from $G$, a total of $d_{1}+d_{2}+\cdots+d_{k}$ edges are also deleted giving that $\bar{m}=m-\left(d_{1}+d_{2}+\cdots+d_{k}\right)$. It proves the result.

Finally, if some pairs of the deleted vertices are adjacent, then we would have the following result.

Theorem 3. If $t$ pairs of the vertices $v_{1}, v_{2}, \ldots, v_{k}$ are adjacent in $G$, then

$$
\bar{d}_{G-\left\{v_{1}, v_{2}, \ldots, v_{k}\right\}}=\frac{2 m-2\left(d_{1}+d_{2}+\cdots+d_{k}\right)+2 t}{n-k} .
$$

Proof. The proof is quite similar to the one of Theorem 2, except we need to deal with adjacent pairs of vertices. If $v_{i}$ and $v_{j}$ are two adjacent vertices in $G$, then the total number of neighbours of $v_{i}$ is $d_{i}$ and the total number of neighbours of $v_{j}$ is $d_{j}$. Hence, when deleting both $v_{i}$ and $v_{j}$, we also delete $d_{i}+d_{j}-1$ edges incident to at least one of $v_{i}$ or $v_{j}$. Here, -1 comes as the edge between $v_{i}$ and $v_{j}$ which can only be deleted once, but it is incident to both $v_{i}$ and $v_{j}$. So, when $v_{1}, \ldots, v_{k}$ are deleted from $G$, a total of $2\left(d_{1}+d_{2}+\cdots+\right.$ $\left.d_{k}-t\right)$ edges are also deleted, giving the result.

Example 1. Let $G$ be the graph in Figure 1, and let us delete $v_{1}, v_{2}, v_{4}, v_{5}$, and $v_{6}$.

Note that the average degree of $G$ is $14 / 7=2$. The graph $G-\left\{v_{1}, v_{2}, v_{4}, v_{5}, v_{6}\right\}$ is in Figure 2 and has the average degree $2 / 2=1$.

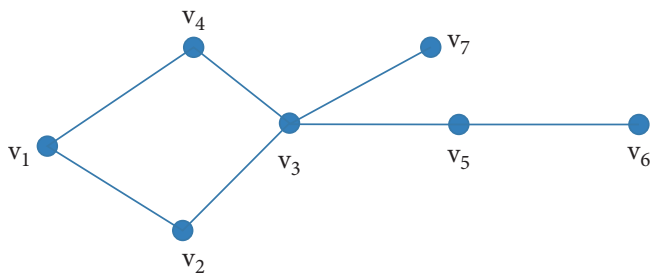

Figure 1: A graph G.

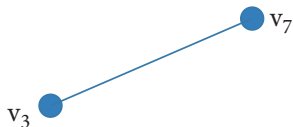

Figure 2: The graph $G-\left\{v_{1}, v_{2}, v_{4}, v_{5}, v_{6}\right\}$.

According to Theorem 3,

$$
1=\frac{2 \cdot 7-2(2+2+2+2+1)+6}{7-5}
$$

as there are $t=3$ pairs of deleted vertices.

\section{Effect of Edge Deletion on Average Degree}

We now determine the effect of edge deletion from a graph on the average vertex degree. Let $G$ be a graph of order $n$ and size $m$, and let $e$ be an edge. The graph obtained by deleting the edge $e$ from $G$ is denoted by $G-e$. We have the following recurrence relation between the average degrees of $G$ and $G-e$.

Theorem 4. The average degree of $G-e$ is related to the average degree of $G$ by the following recurrence relation:

$$
\overline{d_{G}}-\bar{d}_{G-e}=\frac{2}{n} \text {. }
$$

Proof. As deleting an edge $e$ will reduce the number of edges by 1 and will not change the number of vertices, we know that

$$
\begin{aligned}
\bar{d}_{G-e} & =\frac{\sum_{u \in V(G)} d_{G}(u)-2}{n} \\
& =\frac{n \cdot \overline{d_{G}}-2}{n},
\end{aligned}
$$

giving the required result.

\section{Average Degree of Some Derived Graphs}

A derived graph is a graph obtained from a given graph after some operation. In that sense, many authors consider derived graphs as graph operations. Derived graphs help to determine a property of a given graph by calculating the same property of the derived graph. In this section, we determine the average degree of some derived graphs. The derived graphs under study are the line, total, jump, and semitotal line graphs. 
The line graph $L(G)$ of a graph $G$ is constructed as follows. For each edge in the graph $G$, we draw a new vertex of $L(G)$ so that two edges in $G$, having a vertex in common, make an edge between their corresponding vertices in $L(G)$. It can be seen that the order and size of the line graph $L(G)$ are $n(L(G))=m(G)=m$ and $m(L(G))=\left(\left(M_{1}(G)\right) / 2\right)$ $-m$, respectively, where $M_{1}(G)=\sum_{u \in V(G)} d_{G}(u)^{2}$ is the famous topological graph index called the first Zagreb index. Indeed,

$$
\begin{aligned}
m(L(G)) & =\frac{1}{2} \sum_{e \in E(G)} d_{G}(e) \\
& =\frac{1}{2} \sum_{e=u v \in E(G)}\left(d_{G}(u)+d_{G}(v)-2\right) \\
& =\frac{1}{2} \sum_{u \in V(G)} d_{G}(u)^{2}-\sum_{e \in E(G)} 1 \\
& =\frac{M_{1}(G)}{2}-m .
\end{aligned}
$$

Hence, the average degree of the line graph $L(G)$ is obtained as

$$
\bar{d}_{L(G)}=\frac{M_{1}(G)-2 m(G)}{m(G)}=\frac{M_{1}(G)}{m}-2 .
$$

The total graph $T(G)$ of a graph $G$, also known as the generalization of the line graph, is the graph so that the vertex set of $T(G)$ corresponds to the vertices and edges of $G$ and two vertices are adjacent in $T(G)$ iff their corresponding elements are either adjacent or incident in $G$. By the definition, one can deduce the order and size of the total graph $T(G)$ as $n(T(G))=m+n$ and $m(T(G))=2 m+\left(M_{1}(G) / 2\right)$, respectively. Hence, we deduce the average degree of the total graph as

$$
\bar{d}_{T(G)}=\frac{4 m+M_{1}(G)}{m+n} .
$$

Next, we study the average degree of the jump graph. The jump graph $J(G)$ of a graph $G$ is the complement of the line graph. That is, the jump graph $J(G)$ of a graph $G$ is the graph defined on the edge set $E(G)$ of the graph $G$ in which two vertices are adjacent if and only if they are not adjacent in $G$. The order and size of the jump graph are $n(J(G))=m$ and $m(J(G))=((m(m+1)) / 2)-(1 / 2) M_{1}(G)$, respectively. Hence, the average degree of $J(G)$ is

$$
\begin{aligned}
\bar{d}_{J(G)} & =\frac{m^{2}+m-M_{1}(G)}{m} \\
& =m+1-\frac{M_{1}(G)}{m} .
\end{aligned}
$$

Finally, we consider the semitotal line graph $T_{1}(G)$. Its vertex set is $V(G) \cup E(G)$ where two vertices are adjacent in $T_{1}(G)$ if and only if they are either adjacent or incident in $G$. Also, $n\left(T_{1}(G)\right)=m+n$ and $m\left(T_{1}(G)\right)=m+\left(M_{1}(G) / 2\right)$, implying that

$$
\bar{d}_{T_{1}(G)}=\frac{2 m+M_{1}(G)}{m+n} .
$$

So, it is proved.

Theorem 5. Let $G$ be a graph of order $n$ and size $m$. Then, the average degree of the line graph $L(G)$, total graph $T(G)$, jump graph $J(G)$, and semitotal line graph $T_{1}(G)$ is as follows:

$$
\begin{aligned}
& \bar{d}_{L(G)}=\frac{M_{1}(G)-2 m(G)}{m(G)}=\frac{M_{1}(G)}{m}-2, \\
& \bar{d}_{T(G)}=\frac{4 m+M_{1}(G)}{m+n}, \\
& \bar{d}_{J(G)}=m+1-\frac{M_{1}(G)}{m} \\
& \bar{d}_{T_{1}(G)}=\frac{2 m+M_{1}(G)}{m+n} .
\end{aligned}
$$

\section{Average Degree of Some Graph Operations}

Let $G_{1}=\left(V_{1}, E_{1}\right)$ and $G_{2}=\left(V_{2}, E_{2}\right)$ be two graphs such that $\left|E\left(G_{1}\right)\right|=m_{1}$ and $\left|E\left(G_{2}\right)\right|=m_{2}$. In this final section, we study the average degree of some graph operations, namely, union, join, and corona products of two graphs $G_{1}$ and $G_{2}$.

The union $G_{1} \cup G_{2}$ of two graphs $G_{1}$ and $G_{2}$ having disjoint vertex and edge sets is obtained easily by taking union of vertex sets as its vertex set and the union of edge sets as the edge set.

Theorem 6. The average degree of the union $G_{1} \cup G_{2}$ of two graphs $G_{1}$ and $G_{2}$ is

$$
\bar{d}_{G_{1} \cup G_{2}}=\frac{2\left(m_{1}+m_{2}\right)}{n_{1}+n_{2}} .
$$

Proof. By the definition of union operation, we can write that $\left|V\left(G_{1} \cup G_{2}\right)\right|=n_{1}+n_{2}$ and $\left|E\left(G_{1} \vee G_{2}\right)\right|=m_{1}+m_{2}$. This proves the result.

For two graphs $G_{1}$ and $G_{2}$ with orders $n_{1}$ and $n_{2}$ and sizes $m_{1}$ and $m_{2}$, respectively, the join operation $G_{1} \vee G_{2}$ of two graphs $G_{1}$ and $G_{2}$ having disjoint vertex sets $V\left(G_{1}\right)$ and $V\left(G_{2}\right)$ and edge sets $E\left(G_{1}\right)$ and $E\left(G_{2}\right)$ is the graph union $G_{1} \cup G_{2}$ together with all the edges between $V\left(G_{1}\right)$ and $V\left(G_{2}\right)$.

Theorem 7. The average degree of the join $G_{1} \vee G_{2}$ of two graphs $G_{1}$ and $G_{2}$ is

$$
\bar{d}_{G_{1} \vee G_{2}}=\frac{2\left(m_{1}+m_{2}+n_{1} n_{2}\right)}{n_{1}+n_{2}} .
$$

Proof. By the definition, we can deduce that $\left|V\left(G_{1} \vee G_{2}\right)\right|=$ $n_{1}+n_{2}$ and $\left|E\left(G_{1} \vee G_{2}\right)\right|=m_{1}+m_{2}+n_{1} n_{2}$. Hence, the result follows. 
The corona product $G_{1}{ }^{\circ} G_{2}$ of two graphs $G_{1}$ and $G_{2}$ is defined to be the graph obtained by taking one copy of $G_{1}$ (which has $n_{1}$ vertices) and $n_{1}$ copies of $G_{2}$, and then, joining the $i$ th vertex of $G_{1}$ to every vertex in the $i$ th copy of $G_{2}$, for $i=1,2, \ldots, n_{1}$, we obtain the following.

Theorem 8. The average degree of the corona product $G_{1}{ }^{\circ} G_{2}$ of two graphs $G_{1}$ and $G_{2}$ is

$$
\bar{d}_{G_{1} \circ \mathrm{G}_{2}}=\frac{2\left(m_{1}+n_{1}\left(m_{2}+n_{2}\right)\right)}{n_{1}\left(1+n_{2}\right)} \text {. }
$$

Proof. We know that $\left|V\left(G_{1}^{\circ} \mathrm{G}_{2}\right)\right|=\mathrm{n}_{1}\left(1+\mathrm{n}_{2}\right)$ and $\left|E\left(G_{1}^{\circ} G_{2}\right)\right|=m_{1}+n_{1}\left(m_{2}+n_{2} \lim _{x \rightarrow \infty}\right)$. This gives the required number.

\section{Data Availability}

No data available.

\section{Conflicts of Interest}

The authors declare that they have no conflicts of interest.

\section{References}

[1] S. Delen and I. Naci Cangul, "A new graph invariant," TJANT, vol. 6 , no. 1 , pp. $30-33,2018$.

[2] S. Delen and I. N. Cangul, "Extremal problems on components and loops in graphs," Acta Mathematica Sinica, English Series, vol. 35, no. 2, pp. 161-171, 2019.

[3] F. K. Bell, "A note on the irregularity of graphs," Linear Algebra and Its Applications, vol. 161, pp. 45-54, 1992.

[4] H. Abdo, S. Brandt, and D. Dimitrov, "The total irregularity of a graph," Discrete Mathematics \& Theoretical Computer Science, vol. 16, pp. 201-206, 2014.

[5] L. Von Collatz and U. Sinogowitz, "Spektren endlicher Grafen," Abhandlungen aus dem Mathematischen Seminar der Universität Hamburg, vol. 21, pp. 63-77, 1957.

[6] A. Ali and T. Reti, "Two irregularity measures possessing high discriminatory ability," Contributions to Mathematics, vol. 1, pp. 27-34, 2020 . 